\title{
RESEARCH
}

Open Access

\section{circFOXM1 promotes proliferation of non- small cell lung carcinoma cells by acting as a ceRNA to upregulate FAM83D}

Chengtao $\mathrm{Yu}^{1 \dagger}$, Zhuoan Cheng ${ }^{1 \dagger}$, Shaohua Cui ${ }^{2 \dagger}$, Xiaowei Mao ${ }^{2}$, Botai $\mathrm{Li}^{1}$, Yujie Fu ${ }^{3}$, Hui Wang ${ }^{4}$, Haojie $\mathrm{Jin}^{4}$, Qing Ye ${ }^{3}$, Xiaojing Zhao ${ }^{3}$, Liyan Jiang ${ }^{2^{*}}$ and Wenxin Qin ${ }^{14^{*}}$

\begin{abstract}
Background: Biological role and clinical significance of circular RNAs (circRNAs) remain largely unknown. Herein, we aimed to investigate biological function, molecular mechanism, and clinical significance of a circular RNA FOXM1 (circFOXM1) in non-small cell lung cancer (NSCLC).

Methods: Expression of circFOXM1 was measured in 48 paired samples of NSCLC by qRT-PCR. Functional roles of circFOXM1 on tumor cells were explored by in vitro and in vivo assays. Transcriptome sequencing was employed to screen the molecules involved in circFOXM1 regulatory network. RNA immunoprecipitation, luciferase analysis, RNA pull-down, and rescue assay were used to investigate potential mechanisms of circFOXM1.

Results: We found that circFOXM1 was significantly upregulated in NSCLC tissues, and its upregulation was positively correlated with advanced clinical stage and poor prognosis of NSCLC patients. Gain or loss-of-function assay showed that circFOXM1 promoted cell proliferation and cell cycle progression. In vivo assays showed that silencing circFOXM1 inhibited xenograft tumor growth. Mechanically, transcriptome sequencing data indicated that silencing circFOXM1 led to the downregulation of cell cycle-related mRNAs. RNA pull-down and dual-luciferase reporter assay suggested that circFOXM1 could bind to miR-614, and FAM83D was an essential gene involved in the circFOXM1/miR-614 regulatory network.

Conclusions: circFOXM1 promotes NSCLC progression by interacting with miR-614 and thus inactivating the function of miR-614, which will further release the suppression of FAM83D. circFOXM1/miR-614/FAM83D regulatory network may serve as a potential therapeutic target for NSCLC patients.
\end{abstract}

Keywords: circFOXM1, Non-small cell lung cancer, miR-614, FAM83D

\footnotetext{
*Correspondence: jiang_liyan2000@126.com; wxqin@sjtu.edu.cn

${ }^{\dagger}$ Chengtao Yu, Zhuoan Cheng and Shaohua Cui contributed equally to this work.

${ }^{2}$ Department of Respiratory Medicine, Shanghai Chest Hospital, Shanghai Jiao Tong University, Shanghai 200030, China

'State Key Laboratory of Oncogenes and Related Genes, Shanghai Cancer Institute, Renji Hospital, Shanghai Jiao Tong University School of Biomedical Engineering, Shanghai 200032, China

Full list of author information is available at the end of the article
}

(c) The Author(s). 2020 Open Access This article is licensed under a Creative Commons Attribution 4.0 International License, which permits use, sharing, adaptation, distribution and reproduction in any medium or format, as long as you give appropriate credit to the original author(s) and the source, provide a link to the Creative Commons licence, and indicate if changes were made. The images or other third party material in this article are included in the article's Creative Commons licence, unless indicated otherwise in a credit line to the material. If material is not included in the article's Creative Commons licence and your intended use is not permitted by statutory regulation or exceeds the permitted use, you will need to obtain permission directly from the copyright holder. To view a copy of this licence, visit http://creativecommons.org/licenses/by/4.0/ The Creative Commons Public Domain Dedication waiver (http://creativecommons.org/publicdomain/zero/1.0/) applies to the data made available in this article, unless otherwise stated in a credit line to the data. 


\section{Background}

Lung cancer is the leading cause of cancer-related deaths worldwide. Non-small cell lung cancer (NSCLC) is primary histological subtype and accounts for $\sim 85 \%$ of lung cancers, including two major histological types, lung adenocarcinoma and lung squamous cell carcinoma $[1,2]$. Recently, although the treatment of advanced NSCLC has made significant progress by targeted therapies and immunotherapies, five-year survival rate of NSCLC patients is still less than 15\% [3]. One of the major challenges is to find genes essential for NSCLC progression and understand the mechanisms of them [4, 5]. In the past few decades, the investigation into the NSCLC has typically focused on protein-coding genes. However, the limitation of NSCLC therapy is still not fully overcome. Considering that the protein-coding gene accounts for only about $2 \%$ of the whole transcriptome and the remaining $98 \%$ is transcribed into noncoding RNAs (ncRNAs) [6], it is critical to examine the potential of ncRNAs and comprehend the mechanisms underlying the progression of NSCLC.

Circular RNAs (circRNAs) constitute a novel class of ncRNAs. Pervasive expression of circRNAs is a recently discovered feature in various kinds of eukaryotes, including human cells $[7,8]$. circRNAs are usually considered as a master regulator of cellular processes due to their unique properties, such as abundant, stable, cell- and tissue specific expressions [9]. An increasing number of studies have revealed that circRNAs are correlated with clinical features of NSCLC patients and play as regulators in NSCLC. For example, circTP63 is positively correlated with tumor size and TNM stage, exerting oncogenic potential by sponging to miR-873 in lung squamous cell carcinoma [10]. Another circRNA, circPRKCI, also plays as an oncogene, and its therapeutic potential is confirmed in the nude mouse xenograft model and patient-derived tumor xenografts model. circPRKCI acts as a sponge for both miR-545 and miR-589 and abolishes their suppression on the oncogene E2F7 [11]. However, only a small fraction of circRNAs in the genome has been investigated until now, thus further insight into molecular mechanisms and clinical relevance of more functional circRNAs may facilitate the identification of potential biomarkers and therapeutic targets for NSCLC.

Family with sequence similarity 83-member D (FAM83D), a microtubule-associated protein, has been implicated as an oncogene in the various kinds of malignancy. Wang et al. have shown that both of the mRNA and protein levels of FAM83D are upregulated in gastric cancer, and high expression of FAM83D predicts worse overall survival and disease-free survival [12]. Liao et al. report that FAM83D is elevated in hepatocellular carcinoma, and the upregulation of FAM83D significantly promotes cell proliferation and invasion [13]. Furthermore, Yan et al. and $\mathrm{He}$ et al. demonstrate that FAM83D is regulated by miR-495 and miR-210 [14, 15]. Cyclin D1 (CCND1) and cyclin E1 (CCNE1) are two essential downstream mediators of FAM83D [16].

In this study, we explored the role of circFOXM1(has circ_0025039), a circular RNA obviously upregulated in our previous microarray results (GSE126533). We first identified the circular form of circFOXM1 and then verified the upregulated expression in NSCLC tissues compared to adjacent normal tissues. circFOXM1 was correlated with advanced clinical stage and worse overall survival in NSCLC patients. In vitro and in vivo assays showed that circFOXM1 promoted cell proliferation and cell cycle progression. RNA-sequencing results suggested that cell-cycle related genes were modulated by circFOXM1. circFOXM1 functioned as a ceRNA to sponge miR-614 and upregulated FAM83D level. circFOXM1/ miR-614/FAM83D regulatory network may play essential roles in cell growth of NSCLC.

\section{Materials and methods}

\section{Tissues and cell lines}

48 paired samples of tumor tissues( $\mathrm{T}$ ) and their corresponding nontumor tissues $(\mathrm{N})$ from patients with NSCLC were obtained from the Shanghai Chest Hospital, Shanghai Jiao Tong University (Shanghai, China). The NSCLC tissues were immediately stored in liquid nitrogen when they were removed from patients in surgery. The tissues were transported to sample bank of Shanghai Chest hospital and stored in liquid nitrogen. Subsequently, RNAs were extracted from tissues and only qualified RNAs were retained for study. An apart of qualified RNAs was used as template for cDNA reverse transcription. The RNAs and cDNAs were divided into different tubes (RNase-free and DNase-free) to avoid freeze and thaw repeatedly. The qualified RNAs and cDNAs were stored at $-80^{\circ} \mathrm{C}$ until use. These samples were identified by two pathologists independently. The detailed clinicopathological features were described in Table 1. All tissues were collected from July 2013 to September 2014. Written consent approving the usage of tissues in our study was obtained from each patient. This study was approved by the Ethics Committees of the Shanghai Chest Hospital, Shanghai Jiao Tong University. Human NSCLC cell lines, including H1299, $\mathrm{H} 2170$, A549, and H1703, as well as BEAS-2B were purchased from the American Type Culture Collection (ATCC) and were tested negative for mycoplasma contamination. H1299, H2170, A549, and H1703 cells were cultured in RPMI 1640 medium (Life Technologies). BEAS-2B cells were cultured in MEM medium (Life Technologies). All medium was supplemented with $10 \%$ fetal bovine serum and 100 units $/ \mathrm{mL}$ penicillin-streptomycin 
Table 1 Correlations between circFOXM1 and clinicopathological parameters of NSCLC tissues

\begin{tabular}{|c|c|c|c|c|}
\hline \multirow{2}{*}{$\begin{array}{l}\text { Clinicopathological } \\
\text { parameters }\end{array}$} & \multirow{2}{*}{$\begin{array}{l}\text { No. } \\
\text { of } \\
\text { cases }\end{array}$} & \multicolumn{2}{|c|}{ Relative expression } & \multirow{2}{*}{$\begin{array}{l}P \\
\text { value }\end{array}$} \\
\hline & & High & Low & \\
\hline \multicolumn{5}{|l|}{ Gender } \\
\hline male & 41 & 22 & 19 & 0.219 \\
\hline female & 7 & 2 & 5 & \\
\hline \multicolumn{5}{|l|}{ Age } \\
\hline$\geq 60$ & 33 & 17 & 16 & 0.755 \\
\hline$<60$ & 15 & 7 & 8 & \\
\hline \multicolumn{5}{|l|}{ Tumor size } \\
\hline$\geq 4$ & 25 & 18 & 7 & $0.001^{* *}$ \\
\hline$<4$ & 23 & 6 & 17 & \\
\hline \multicolumn{5}{|c|}{ Lymphatic metastasis } \\
\hline positive & 27 & 15 & 12 & 0.382 \\
\hline negative & 21 & 9 & 12 & \\
\hline \multicolumn{5}{|l|}{ History type } \\
\hline adenocarcinoma & 15 & 6 & 9 & 0.459 \\
\hline squamous & 33 & 16 & 17 & \\
\hline \multicolumn{5}{|l|}{ TNM stage } \\
\hline$|/| \mid$ & 25 & 9 & 16 & $0.043^{*}$ \\
\hline III/IV & 23 & 15 & 8 & \\
\hline
\end{tabular}

${ }^{*} P<0.05$ ** $P<0.01$

(Life Technologies). All cells were maintained in humidified incubator at $37^{\circ} \mathrm{C}$ in a $\mathrm{CO}_{2}$ incubator.

\section{RNA and DNA extraction}

Total RNAs of tissues and cells were extracted by using Trizol reagent (Invitrogen). All experiment operations were followed the manufacturer's instruction of Trizol reagent. The procedure of RNAs extracted from nuclear fractions or cytoplasmic fractions were according to PARIS Kit (Life Technologies) manufacturer's protocol. For DNA extraction, cells were rinsed with PBS twice and then extracted by Genomic DNA Isolation Kit (Sangon Biotech, China).

\section{RNase $\mathrm{R}$ treatment}

RNase R (Epicentre Technologies) was used to treat with total RNAs. Briefly, extracted RNAs aliquots from H1299 and H2170 cells were split into two parts: one for RNase $\mathrm{R}$ digestion and another for control with digestion buffer only. For RNase $\mathrm{R}$ digestion, $2 \mu \mathrm{g}$ of total RNA was mixed with $2 \mu \mathrm{l} 10 \times$ RNase R Reaction Buffer and $2 \mu \mathrm{l}$ RNase $\mathrm{R}(20 \mathrm{U} / \mu \mathrm{l})$; for control, RNase R was replaced with DEPC-treated water. Then, the RNA samples were incubated at $37^{\circ} \mathrm{C}$ water bath heater for 30 min. The detection of circFOXM1 and FOXM1 mRNA was analyzed by PCR, RT-PCR or qRT-PCR. RNase R treated RNA was used only for detecting resistance of circFOXM1 to RNase $\mathrm{R}$ exonuclease digestion. All primers were listed in Additional file 1: Table S1.

\section{Reverse transcription PCR(RT-PCR) and quantitative real- time PCR (qRT-PCR)}

For RT-PCR, 500 ng RNA was treated with gDNA wiper for $2 \mathrm{~min}$ at $42^{\circ} \mathrm{C}$ and then was used to synthesize cDNA by using Hiscript Revert 1st First Strand cDNA Synthesis Kit (Vazyme, China). cDNA was used as templates to amplify by DNA Polymerase (Life Technologies), and products were further verified by using $1.5 \%$ agarose gel electrophoresis.

For qRT-PCR, only the cDNA was used as template and qRT-PCR assays were investigated by AceQ qPCR SYBR Green Master Mix (Vazyme, China) kits on ABI 7500 qPCR system. The circRNA and mRNA levels were normalized by $\beta$-actin. miRNA level was normalized by U6. The relative expression levels were determined by the $2^{-\Delta \mathrm{Ct}}$ or $2^{-\Delta \Delta \mathrm{Ct}}$ method. To determine the absolute quantity of RNA, the purified PCR product amplified from cDNA corresponding to the circFOXM1 and FAM83D sequence was serially diluted to generate a standard curve, respectively. Briefly, circFOXM1 and FAM83D form cDNAs were amplified, purified and measured. Then they were serially diluted to be as templates for qRT-PCR. The standard curves were drawn according to the $\mathrm{Ct}$ values at different concentrations. According to the standard curves, copy numbers of circFOXM1 and FAM83D in NSCLC cell lines were calculated.

\section{Plasmid construction and transfection}

To construct circFOXM1 ectopic overexpression plasmid, the sequences of exon 4 and exon 5 in FOXM1(amplified from cDNAs of $\mathrm{H} 1299$ cells) were cloned into pZW-circRNA vector (a gift from Ling-Ling Chen Lab) [17]. To construct circFOXM1 knockdown plasmids (shcircFOXM1), fragments targeting the circFOXM1 junction sites were cloned into pGreenPuro vector (System Biosciences). Likely, fragments targeting FAM83D mRNAs were constructed into pGreenPuro vector to generate FAM83D knockdown plasmids (sh-FAM83D). For dual-luciferase assay, wild-type and mutant fragments of circFOXM1 as well as FAM83D 3' UTR were cloned into pmirGLO vector (Promega) to form luciferase reporter vector. The sequences of primers were listed in Additional file 1: Table S1.

For pZW-circFOXM1, sh-circFOXM1 or sh-FAM83D transfection, $2 \times 10^{5}$ cells were seed in $60 \mathrm{~mm}$ dishes for $24 \mathrm{~h}$ before transfection. For shRNA-circFOXM1 or shRNA-FAM83D stable cell line construction, $1 \times 10^{5}$ cells were seed in $60 \mathrm{~mm}$ dishes for $24 \mathrm{~h}$ before virus infection. Lentivirus was added into culture medium with 
polybrene, followed by selection with puromycin $(2 \mu \mathrm{g} /$ $\mathrm{ml}$ ) for 2 weeks. For dual-luciferase assay, plasmids with wild-type or mutant fragments were co-transfected with miR-614, respectively.

\section{Transcriptome sequencing}

Total RNAs were isolated from H1299 cells with circFOXM1silencingorcontrol by using TRIzol reagent (Invitrogen) and purified by RNeasy Mini Kit (Qiagen). Transcriptome sequencing was conducted using Illumina HiSeq ${ }^{\text {tm }} 2000$ by Sangon (Sangon Biotech, China). Differently expressed transcripts were selected by |fold change $\mid>2$ and $F D R<0.1$. Results were collected in Additional file 2: Table S2.

\section{Gene set enrichment analysis}

Gene set enrichment analysis (GSEA) software (version 3.0, www.broadinstitute.org/gsea/) was employed to identify gene sets that were significantly overrepresented among genes up- or down-regulated in circFOXM1 silencing cells. Briefly, gene expression profiles were generated from circFOXM1knockdown and control cells by RNA-sequencing. GSEA v3.0 software was used to explore the distribution of members of the gene sets from the MSigDB database. In this bioinformatics analysis, if the most members in a gene set were positively or negatively correlated with the circFOXM1 expression, the set was termed associated with circFOXM1.

\section{CCK-8 assay}

$1 \times 10^{3}$ cells were seeded in 96-well plates and were cultured for $1 \sim 5$ day, followed by incubation with $10 \mu \mathrm{l}$ of CCK-8 assay solution per well for $2 \mathrm{~h}$ (Dojindo Laboratories, Japan). The absorbance values at $450 \mathrm{~nm}$ were then assessed.

\section{Colony formation assay}

A density of $1 \times 10^{3}$ cells per well were incubated in 6well plates. After 2 weeks' incubation, a total of $1 \%$ of crystal violet (Beyotime Biotechnology, China) was applied to stain cell clones which were fixed with methanol.

\section{Flow cytometry analysis}

After harvesting by trypsinization, cells were washed with pre-cold PBS buffer, and then fixed in $75 \%$ ice-cold ethanol. Before staining, cells were resuspended in cold PBS and subjected to digestion with $2 \mu \mathrm{g} / \mathrm{ml}$ RNase A at $37^{\circ} \mathrm{C}$ for $30 \mathrm{~min}$, followed by labeling with $15 \mu \mathrm{g} / \mathrm{ml}$ propidium iodide (Beyotime Biotechnology, China) for 15 min at room temperature. Cell cycle profiles of labeledcells were analyzed using a FACS Calibur flow cytometer (BD Biosciences).

\section{Fluorescence in situ hybridization (FISH)}

Cells were rinsed by PBS and then fixed in $4 \%$ formaldehyde for $10 \mathrm{~min}$ room temperature. The cells were further permeabilized in PBST (0.5\% Triton X-100) on ice for $10 \mathrm{~min}$, and then prehybridized with prehybridization buffer. After rinsing once in $2 \times \mathrm{SSC}$, hybridization was performed using Cy3-labelled probe at $42^{\circ} \mathrm{C}$ overnight. After co-staining with DAPI, the signals of the probe were captured by confocal microscopy (Zess 7100).

\section{RNA pull-down assay}

For miR-614 pull-down assay, the 3'end biotinylatedmiR614 mimics or miRNA control (Sangon, China) were transfected into cells at a final concentration of $100 \mathrm{nM}$ for $48 \mathrm{~h}$ before harvest. Then IP Cell lysis Buffer (Sangon Biotech, China) and complete protease inhibitor cocktail (Roche Applied Science) were added into the cell pellets, and incubated on ice for $10 \mathrm{~min}$. Biotin-coupled RNA complex was pulled down by incubating the cell lysates with streptavidin-coated magnetic beads (Life Technologies) by centrifugation at $10,000 \times \mathrm{g}$ for $10 \mathrm{~min}$. The abundance of targets was evaluated by qRT-PCR analysis.

For circFOXM1 pull-down assay, $1 \times 10^{7}$ cells that expressed circFOXM1 were harvested, lysed, and sonicated. The biotin-coupled probe of circFOXM1 or probe control was incubated with magnetic beads, respectively. After $2 \mathrm{~h}$ incubation, cell lysates were incubated with the probes overnight at $4{ }^{\circ} \mathrm{C}$. After the incubation, the bound RNAs were washed for six times with wash buffer and purified for the qRT-PCR analysis. The biotinylatedprobe was designed and synthesized by Sangon (Shanghai, China).

\section{Immunoblotting}

Extracted protein samples were quantified and boiled in SDS/ $\beta$-mercaptoethanol buffer, then loaded into polyacrylamide gels. After separation by electrophoresis, the proteins were transferred onto PVDF membranes (Millipore). The membrane was incubated with rabbit antiFAM83D antibody (ab236882, Abcam) or anti-CCND1 antibody (26939-1-AP, proteintech) or anti-CCNE1(115541-AP, proteintech) or anti-FOXM1 antibody (13147-1-AP, proteintech) or anti- $\beta$-actin antibody (20536-1-AP, proteintech) at $4{ }^{\circ} \mathrm{C}$ for $12 \mathrm{~h}$, followed by an incubation with secondary antibody (proteintech) for $1 \mathrm{~h}$. Bands were detected by a Bio-Rad ChemiDoc XRS system.

\section{RNA immunoprecipitation (RIP)}

RIP assays were performed by using a Magna RIP RNABinding Protein Immunoprecipitation Kit (Millipore) according to the protocol. Ago2 antibody was used for RIP (Cell Signaling Technology). Co-precipitated RNA was detected by qRT-PCR. 


\section{Luciferase assay}

Cells were plated onto 96-well plates and grown to 70\% confluence. For circFOXM1 and miR-614 luciferase assay, the cells were co-transfected with $50 \mathrm{nM}$ miR-614 mimics and $200 \mathrm{ng}$ pmirGLO-circFOXM1 vector, or corresponding mutant type (mut). For FAM83D and miR614 luciferase assay, the cells were co-transfected with $50 \mathrm{nM}$ miR-614 mimics and $200 \mathrm{ng}$ pmirGLO-FAM83D vector, or corresponding mutant type (mut). Dual luciferase assay kit (promega) was used for dual luciferase assay. At $48 \mathrm{~h}$ post-transfection, cells were collected, and Renilla luciferase activity was assessed. Results are assessed as the ratio of Firefly luciferase activity to Renilla luciferase activity.

\section{Xenografts experiments}

Five-weeks' old male BALB/c nude mice were chosen and randomly divided into four groups for our experiment. H2170 cells transfected with vector control, pZWcircFOXM1, sh-circFOXM1 or negative control (sh-NC) were subcutaneously injected into the back of the nude mice $\left(1 \times 10^{6}, 100 \mu \mathrm{l}\right)$. The volume and weight of tumors were measured for 35 days. All animal experiments were performed under approval by the Shanghai Medical Experimental Animal Care Commission.

\section{Immunohistochemistry}

Tumor sections were subjected to deparaffinize and rehydrate with gradient ethanol solutions. After being immersed in antigen retrieval solution (AR0023, Boster, China) and heated for $15 \mathrm{~min}$, sections were then incubated with anti-human Ki-67 antibody (M7240, Dako, Denmark) for $1 \mathrm{~h}$ and counterstained with hematoxylin (BA-4226, BASO, China) for $2 \mathrm{~min}$.

\section{Statistical analysis}

Data were expressed as the mean \pm standard deviation from at least three independent experiments. Survival analysis was performed with the Kaplan-Meier method, and the log-rank test was used for comparisons. Statistical results were analyzed using Prism software (GraphPad Software). Student's t-test was used to compare two experimental groups. A probability of 0.05 or less was considered as statistical significance.

\section{Results}

\section{Identification and characteristic of circFOXM1 in NSCLC}

Based on the analysis for our previous microarray data (GSE126533), we found that has_circ_0025039 (chr12: 2975558-2,977,920) was evaluated as an upregulated circRNA in NSCLC (Fig. 1a-b). By browsing the human reference genome (GRCh37/hg19), we knew that has circ_0025039 was derived from the exon 4 and 5 of FOXM1 loci, and thus named it circFOXM1 in this study. Subsequently, we validated the upregulation of circFOXM1 in another 48 paired samples of NSCLC by qRT-PCR. Results showed that the expression of circFOXM1 was significantly higher in $87.5 \%$ (42 of 48) of NSCLC tissues than adjacent nontumor tissues (Fig. 1c-d). Analysis for clinical relevance revealed that the expression level of circFOXM1 was more likely to be positively associated with tumor size and TNM stage (Table 1). In addition, high expression of circFOXM1may predict an unfavorable overall survival of NSCLC patients (Fig. S1A).

To verify that circFOXM1 was circular RNA rather than products of trans-splicing or genomic rearrangements, circRNA identification assays were performed. Firstly, using cDNA or genomic DNA from cells as templates, we found that circFOXM1 was only amplified from cDNA, while no specific amplification product was observed from genomic DNA (Fig. 1e). The back-spliced junction in PCR products of circFOXM1 was also identified by Sanger sequencing (Fig. 1f). Results of resistance to RNase $\mathrm{R}$ exonuclease digestion further confirmed that circFOXM1 was circular in form (Fig. 1g-h). Taken together, these results indicate that circular RNA circFOXM1 is upregulated in NSCLC, and high expression of circFOXM1 is associated with advanced stage and poor survival of NSCLC patients.

circFOXM1 promotes cell proliferation and tumor growth

Next, we measured the expression of circFOXM1in NSCLC cell lines by qRT-PCR. Results showed that circFOXM1 was highly expressed in NSCLC cell lines compared to human normal bronchial epithelium cells (BEAS2B) (Fig. S1B). For constructing ectopic circFOXM1 overexpressing vector, exons 4 and 5 of FOXM1 were cloned into the pZW vectors (Fig. S1C). For silencing circFOXM1, we constructed the short hairpin RNA vector, which specifically targeted the back-splicing junction of circFOXM1(Fig. S1D). As expected, results showed that circFOXM1 was successfully expressed more than 40-fold changes by pZW-circFOXM1 than controls in H1299 and H2170 cells, while FOXM1 mRNA expression had no obvious change (Fig. S1E). Likely, sh-circFOXM1 could successfully decrease the expression of circFOXM1 by about two-fold changes but had no effect on the expression of FOXM1 mRNA (Fig. S1F). Besides, we also found that circFOXM1 had no effect on the level of FOXM1 protein (Fig. S1G-H).

Subsequently, in vitro assays were performed to investigate potential roles of circFOXM1. CCK- 8 results revealed that the viability of both $\mathrm{H} 1299$ and $\mathrm{H} 2170$ cells was increased in circFOXM1 overexpressing group while decreased in circFOXM1 silencing group (Fig. 2a-b). Colony formation assays showed that the ability of colony formation was significantly improved in circFOXM1 overexpressing group, and reduced in circFOXM1 


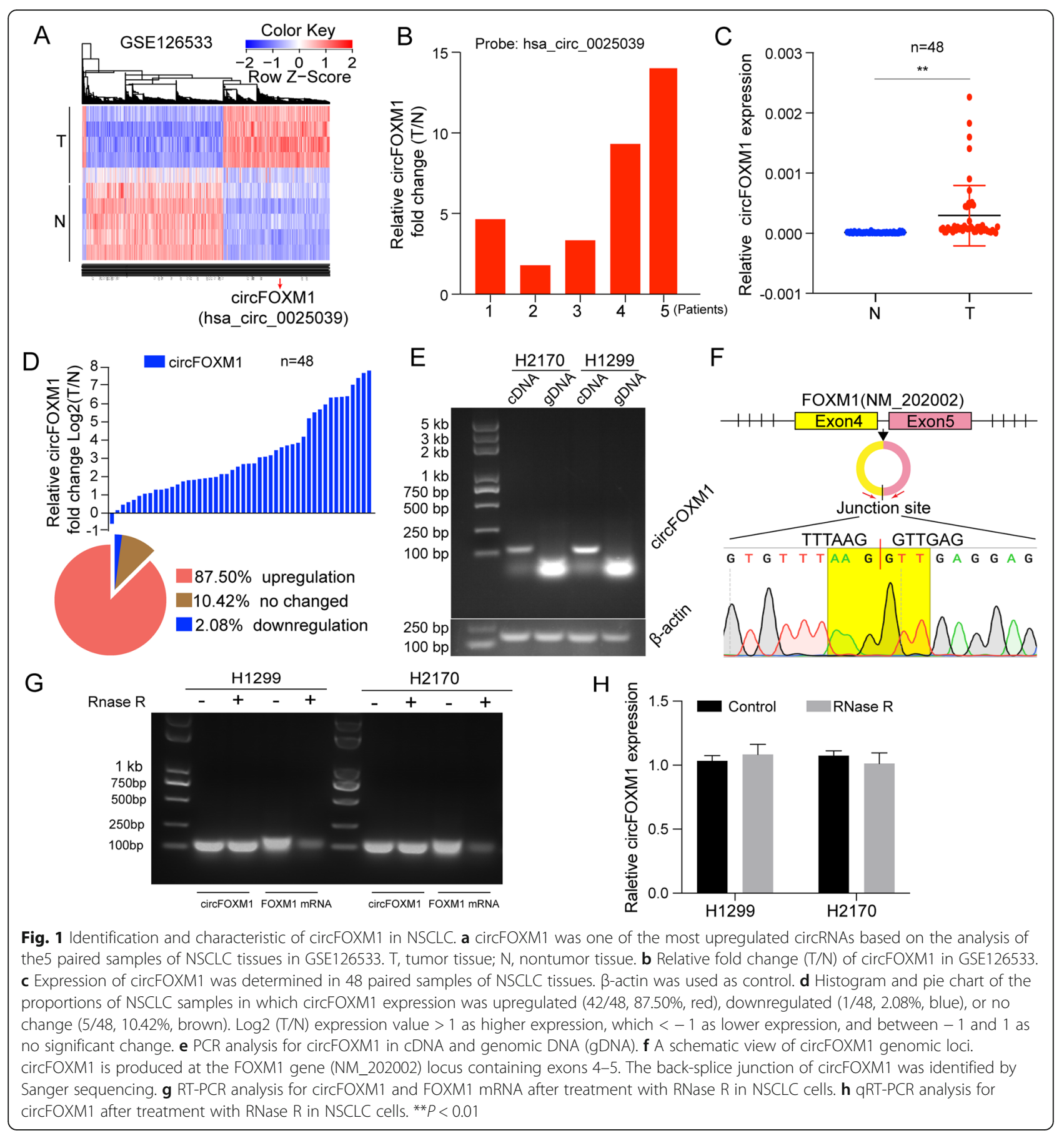

silencing group (Fig. 2c-d). Furthermore, cell cycle analyses showed that the number of cells was increased in G2/M phase when overexpressing circFOXM1. In contrast, silencing circFOXM1 made cells arrested in G0/G1 phase (Fig. 2e-f). These data indicate that circFOXM1 promotes NSCLC cell proliferation and cell cycle progression in vitro.

To identify the effect of circFOXM1 on tumor growth in vivo, we established a nude mice xenograft model by implanting H2170 cells with circFOXM1overexpressing or circFOXM1 silencing. Results showed that tumor cells with circFOXM1overexpressing grew rapidly compared to control. In addition, Ki-67 staining showed that circFOXM1 made more Ki-67 positive cells. In contrast, silencing circFOXM1 obviously blocked upon tumor growth and decreased Ki-67 staining cells (Fig. 2g-j). Taken together, our findings suggest that circFOXM1 may play oncogenic roles in NSCLC progression. 


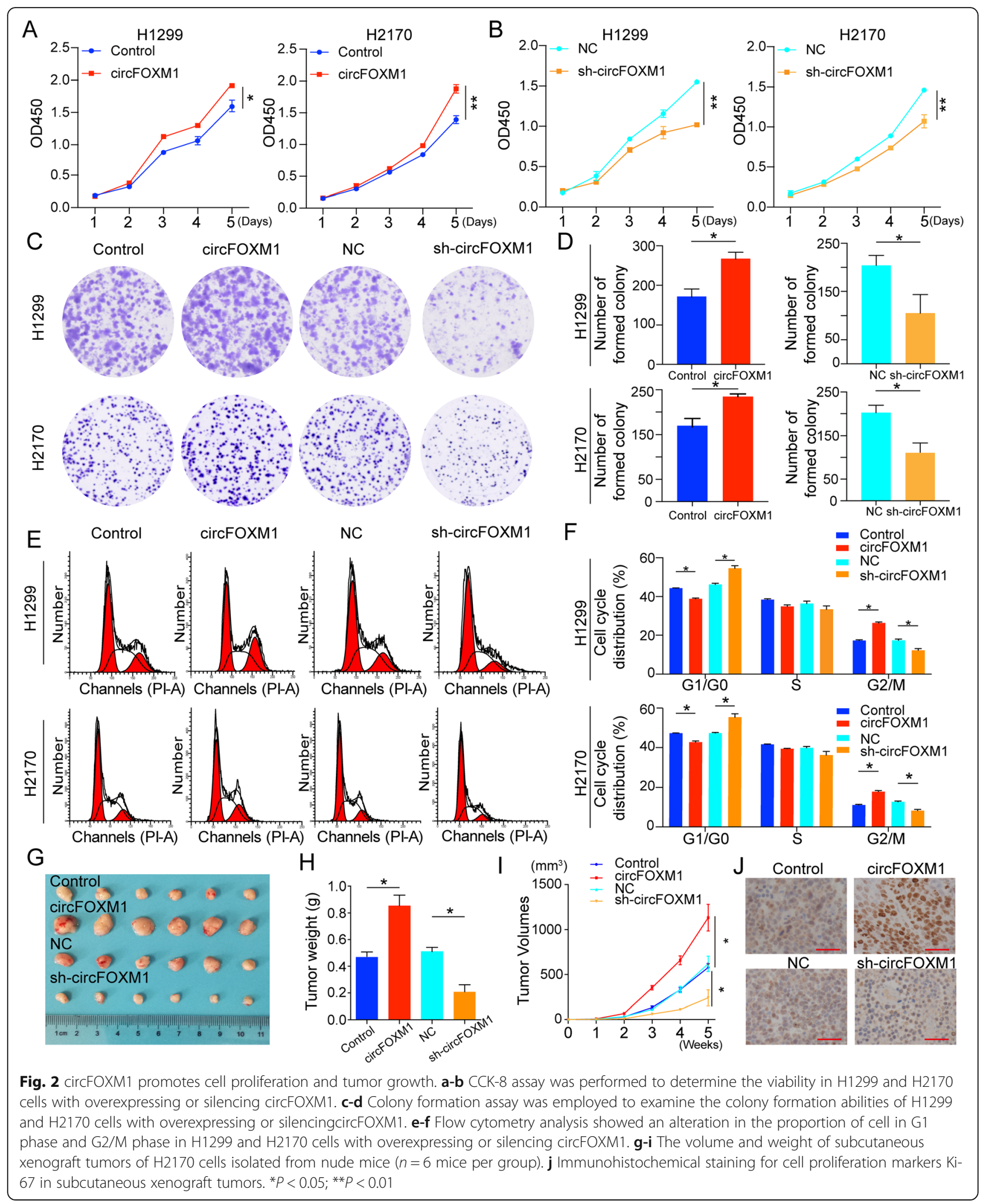




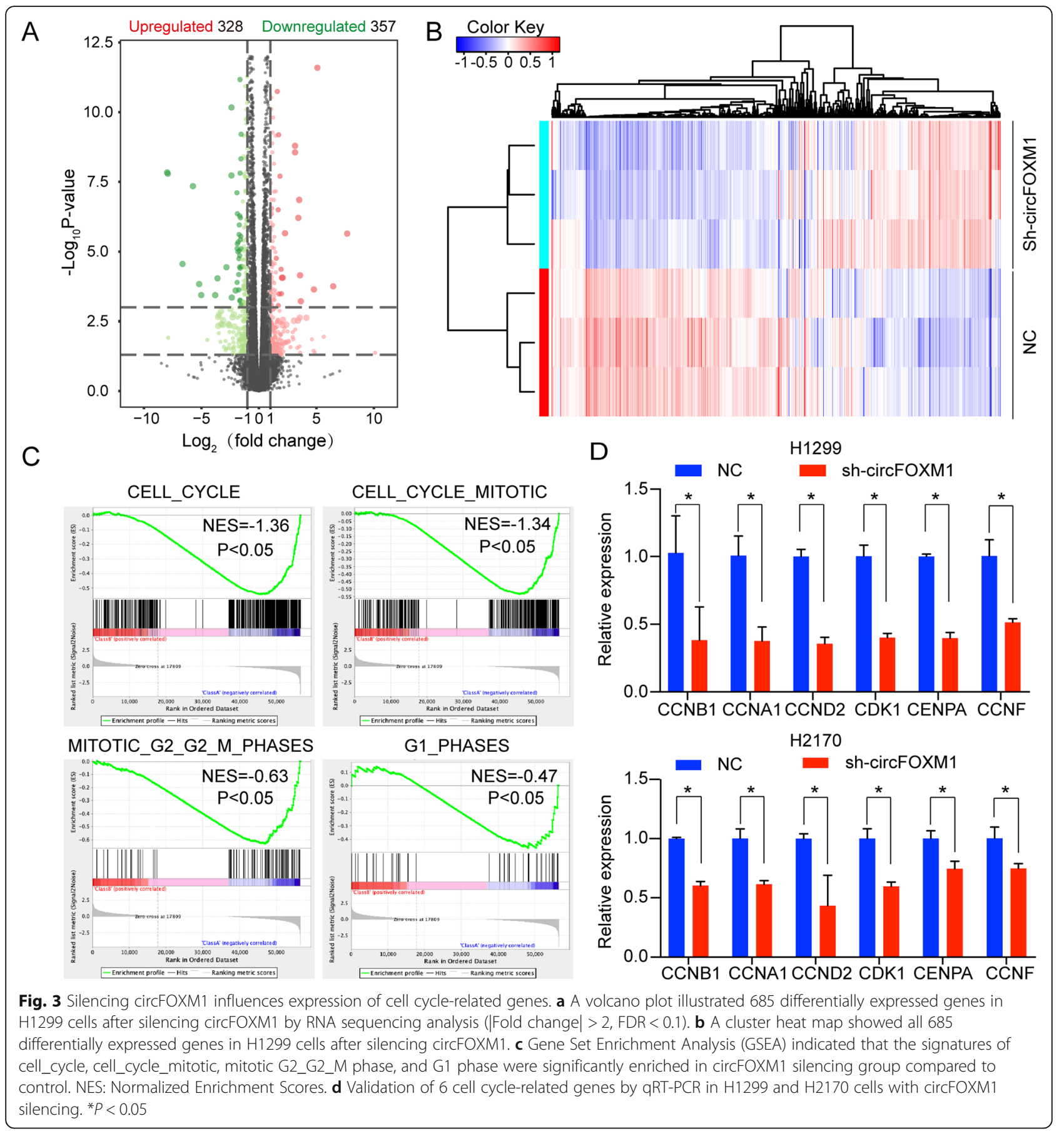

\section{Silencing circFOXM1 influences the expression of cell cycle-related genes}

Using RNA-sequencing technology, we identified 685 dysregulated genes $(\mid$ Fold change $\mid>2$, FDR $<0.1)$ (328 genes upregulated and 357 genes downregulated) after silencing circFOXM1in H1299 cells (Fig. 3a, Additional file 2: Table S2). Hierarchical clustering heat map showed different clustering patterns between circFOXM1 silencing group and control group (Fig. 3b). To further illustrate the mechanism of circFOXM1, we analyzed GSEA results and found that the signatures of cell cycle, cell cycle mitotic, mitotic G2/G2-M phase, and G1 phase were significantly enriched in downregulated genes, indicating that these biologic processes may be suppressed by circFOXM1 silencing (Fig. 3c). Moreover, we tested and verified six genes of these signatures by qRT-PCR. Results showed that the expression of CCNB1, CCNA1, CCND2, CDK1, CENPA, and CCNF were downregulated in both H1299 
and H2170 cells with circFOXM1 silencing (Fig. 3d). These results were consistent with above phenotypes that silencing circFOXM1 made cell cycle arrested in G0/G1 phase. Thus, circFOXM1 may promote cell proliferation by modulating cell cycle-related genes.

\section{circFOXM1 facilitates cell proliferation by sponging miR- 614}

Many studies have revealed that circRNAs play as the major regulators through miRNA sponge, acting as ceRNAs by blocking Ago2-mediated silencing complex [18, 19]. To investigate whether circFOXM1 could function as a ceRNA, we first detected its subcellular localization. As results shown in FISH assay and nuclear/cytoplasmic fraction assay, the majority of circFOXM1 was preferentially localized in the cytoplasm (Fig. 4a-b). RNA-protein immunoprecipitation results revealed that circFOXM1 could bind Ago2 protein (Fig. 4c, Fig. S2A). Thus, we supposed that circFOXM1 had the potential to function as a ceRNA. Then, we determined to screen potential miRNAs binding to circFOXM1 by miRanda prediction (http://159.226.6 7.237:8080/new/analysis_mirna.php), and found that 17 miRNAs (Energy<- 23) were predicted as binding partners with circFOXM1 (Fig. 4d). RNA pull-down assays showed that specific probe against circFOXM1 could enrich circFOXM1 RNAs more than 30-fold changes compared with control (Fig. 4e), and miR-614 was the most enriched miRNA in the captured fraction of circFOXM1 specific probe compared with control in both H2170 and H1299 cells (Fig. 4f-g). To further validate the interaction between circFOXM1 and miR-614, we performed luciferase assays containing wild-type and mutated putative binding sites of circFOXM1 to verify the direct interaction. Our results showed that the luciferase activities of circFOXM1 wild-type reporter were significantly reduced when transfected with miR-614 mimics compared to control or circFOXM1 mutated luciferase reporter. Importantly, cells transfected with plasmids of mutant circFOXM1 could not significantly promote cell proliferation and cell cycle progression (Fig. S2B-C). We then examined the function of miR- 614 by several methods, including CCK- 8 assay, colony formation assay, and cell cycle assay. The results of these assays showed that miR-614 inhibited cell proliferation and cell cycle progression (Fig. S3A-D). Moreover, we also found that miR-614 was downregulated in NSCLC tissues by analyses for our microarray data and qRT-PCR results (Fig. S3E-F). Overall survival analysis in KaplanMeier Plotter database [20] (https://kmplot.com) showed that high expression of miR-614 predicted good prognosis in NSCLC (Fig. S3G). Furthermore, CCK-8 assays revealed that miR-614 could weaken the promotion effect of circFOXM1 in both H1299 and H2170 cells (Fig. 4i-j). Taken together, these results indicate that circFOXM1 promotes cell proliferation mediated by miR- 614 .
miR-614 inhibited cell proliferation via FAM83D

According to ceRNAs theory [19], circFOXM1 should share the miR-614 with mRNA and thus released the repression of mRNA by competitively binding with miR614. Next, we first predicted the targets of miR-614 by Targetscan software(http://www.targetscan.org/vert_72/) [21]. The prediction results showed that $54 \mathrm{mRNAs}$ had the potential to be regulated by miR-614(Additional file 3: Table S3). Then, we analyzed the downregulated RNAs in our RNA-sequencing data after silencing circFOXM1(Additional file 2: Table S2). Only FAM83D was a common target of miR-614 between prediction targets of Targetscan software and downregulated RNAs after silencing circFOXM1(Fig. 5a).Results of western blot and qRT-PCR showed that the protein and mRNA levels of FAM83D were downregulated by miR-614 transfection(Fig. 5b). Luciferase assays containing wild-type and mutated putative binding sites of FAM83D 3'UTR were performed to verify the direct interaction between them. Results showed that the luciferase activities of FAM83D wild-type reporter were significantly reduced when transfected with miR-614 mimics compared to control reporter or FAM83D 3'UTR mutated luciferase reporter (Fig. 5c). RNA pull-down results suggested that miR-614 could enrich FAM83D more than five-fold changes (Fig. 5d). These results indicate that FAM83D is a direct target of miR-614. CCK-8 assays, colony formation assays, and cell cycle assays showed that silencing FAM83D inhibited cell proliferation, colony formation, and cell cycle progression (Fig. S4A-D). In addition, CCK-8 assays showed that overexpression of FAM83D could rescue the inhibitory effects of miR-614 (Fig. 5e-f). We also found that FAM83D was frequently upregulated in NSCLC patients, and high expression of FAM83D predicted poor prognosis [20](Fig. S4E-G). Taken together, these results suggest that FAM83D is a target of miR-614.

\section{circFOXM1 promotes cell proliferation via the miR-614/ FAM83D axis}

qRT-PCR results showed that the copies of circFOXM1 was about 160 and 220 in H1299 and H2170 cells (per 20 pg RNA), while FAM83D were about 220 and 750 in H1299 and H2170 cells (per 20 pg RNA), respectively. These data suggested that expression levels of circFOXM1 and FAM83D were near (Fig. S5). RNA pulldown assays showed that overexpression of circFOXM1 led to less enrichment of FAM83D in miR-614 pulldown assay. However, silencing circFOXM1 caused a significant increase in the recruitment of FAM83D in miR-614 pull-down assay (Fig. 6a-b). Furthermore, results of western blot and qRT-PCR showed that overexpression of circFOXM1 upregulated the mRNA and protein levels of FAM83D, while silencing circFOXM1 


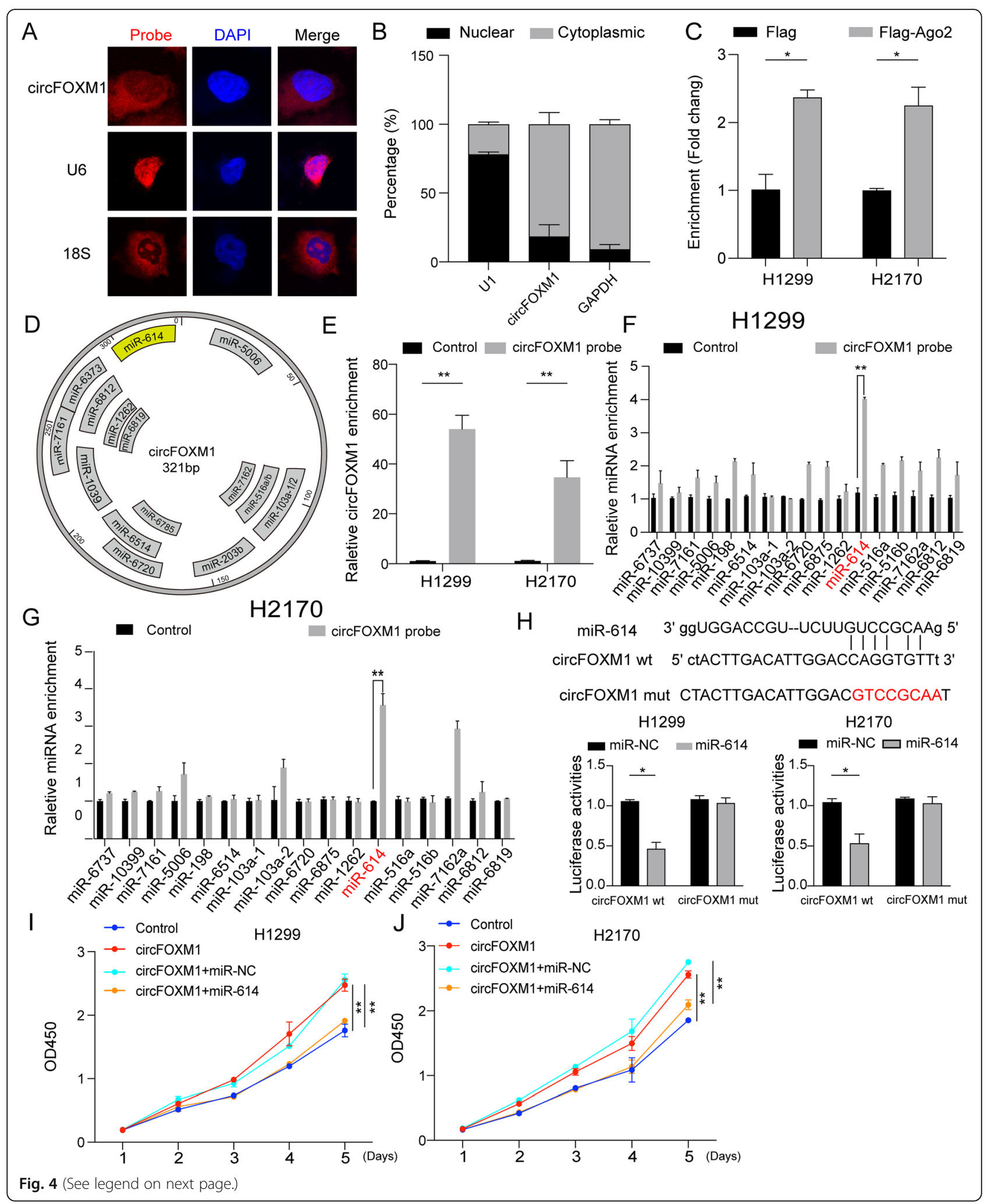


(See figure on previous page.)

Fig. 4 circFOXM1 facilitates cell proliferation by sponging miR-614. a RNA fluorescence in situ hybridization (FISH) showed that circFOXM1 was predominantly localized in cytoplasm in H1299 cells. U6 was mainly localized in nucleus. 185 was mainly localized in cytoplasm. b Cytoplasmic and nuclear RNA fractionation experiments showed that circFOXM1 was mainly located in the cytoplasm in H1299 cells. c Ago2 RIP assay was used for the detection of circFOXM1 in H1299 and H2170 cells expressing Flag-Ago2 or Flag-tag. $\mathbf{d}$ The putative binding sites of 17 predicted miRNAs on circFOXM1. e RNA pull-down assay was used for the detection of circFOXM1 by circFOXM1 specific probe. $\mathbf{f}$-g Relative expression of circFOXM1 putative binding miRNAs was examined by qRT-PCR analysis by circFOXM1 specific probe. $\mathbf{h}$ Luciferase activity of luc-circFOXM1 wt or Luc-circFOXM1 mut in H1299 and H2170 cells co-transfected with miR-614 mimics. i-j Cell proliferation analysis of H1299 and H2170 cells transfected with control, circFOXM1, circFOXM1 + miR-NC (miRNA control), or circFOXM1 + miR-614. ${ }^{*} P<0.05$; ${ }^{* *} P<0.01$
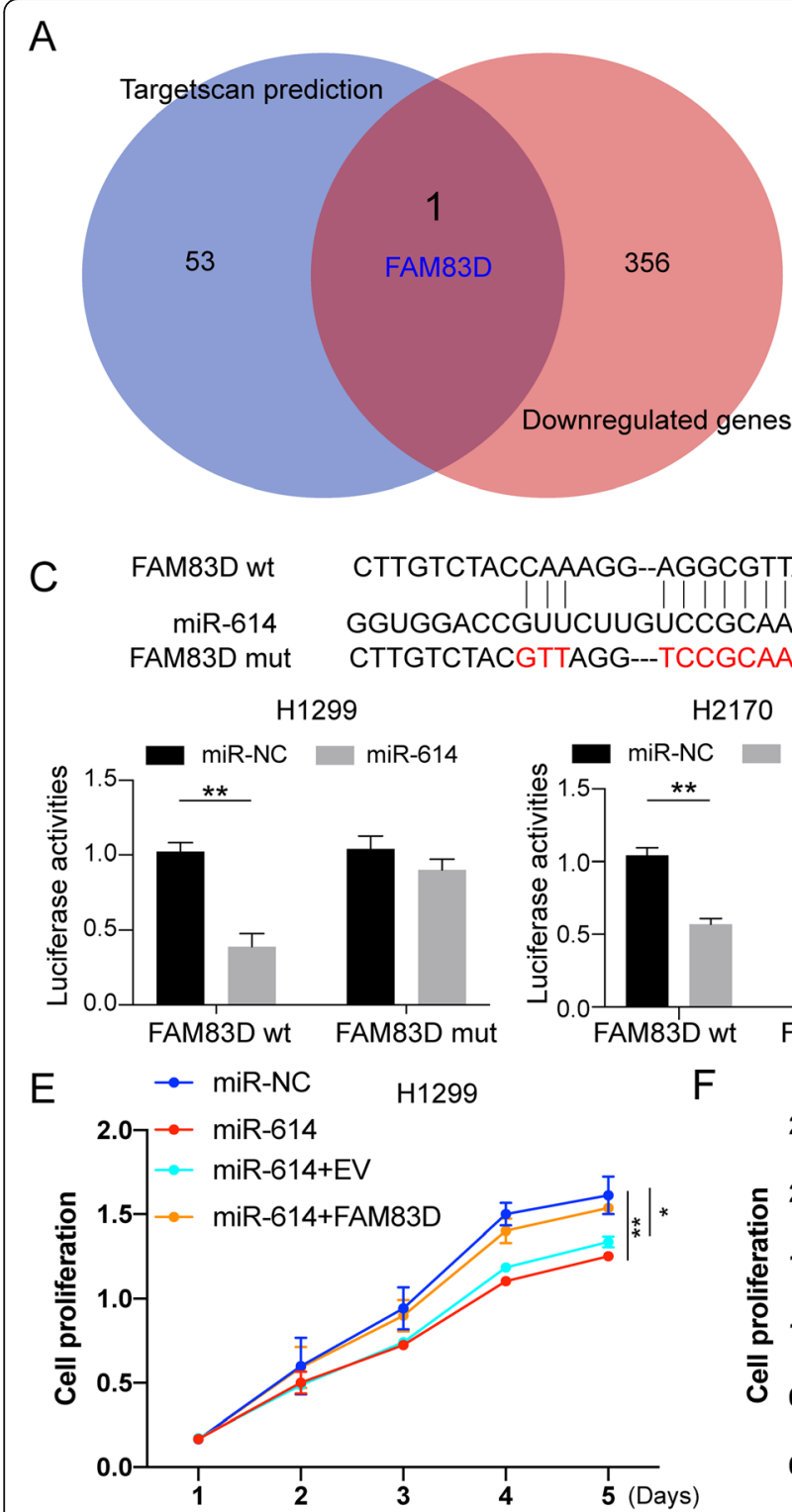

F
B

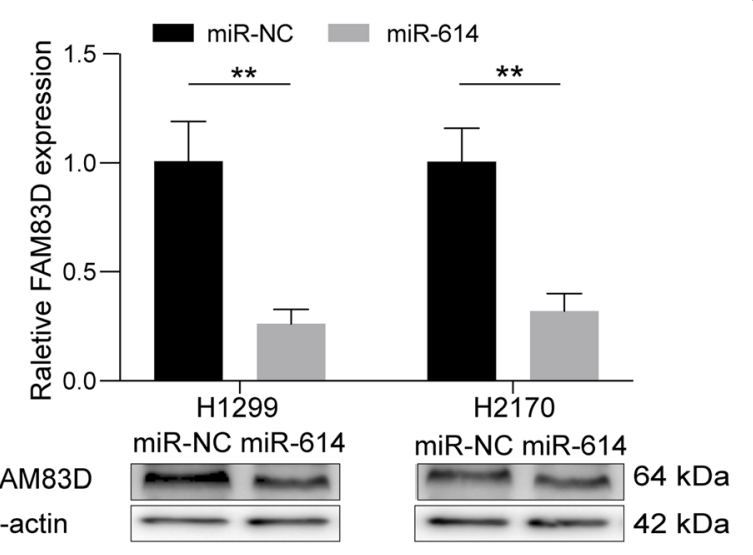

D

bio-miR-NC bio-miR-614
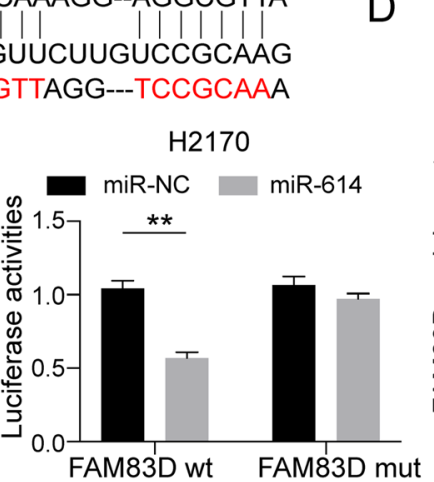
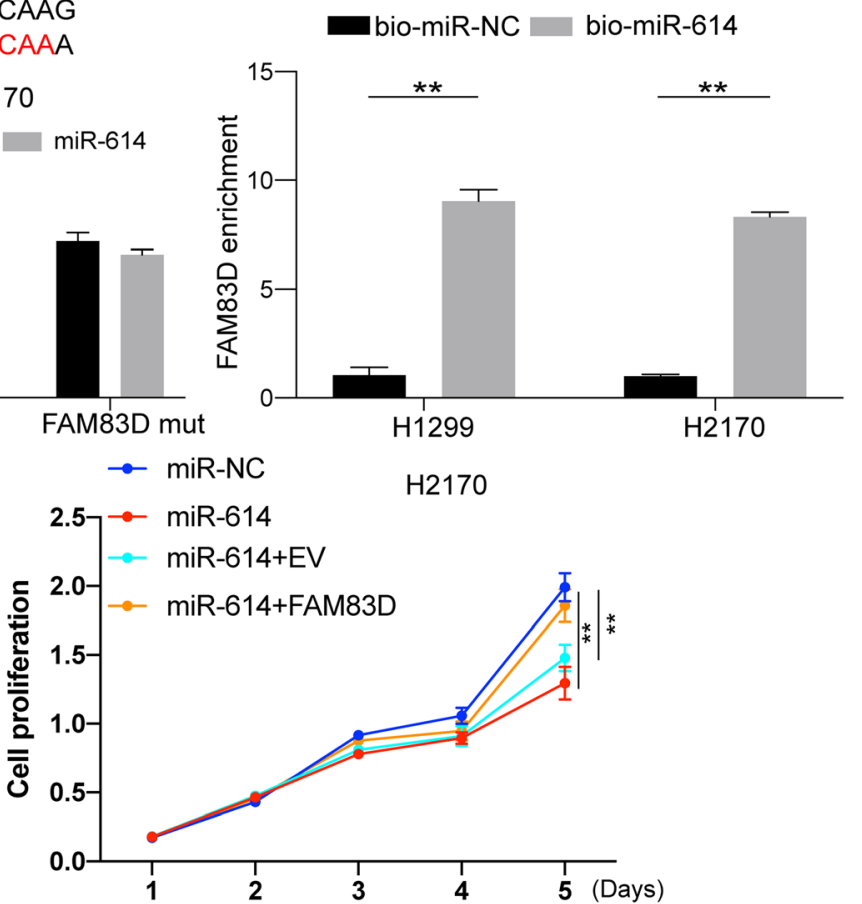

Fig. 5 miR-614 inhibited cell proliferation via FAM83D. a Venn diagram showed the intersection between miR-614 potential targets predicted by Targetscan (blue circle, 8mer sites) and downregulated genes (red circle, ffold change| > 2, FDR < 0.1) after silencing circFOXM1 in H1299 cells. FAM83D is the only gene in the overlapping of the two datasets. $\mathbf{b}$ mRNA and protein levels of FAM83D after transfection of miR-614 or miR-NC in H1299 and H2170 cells. c Upper panel: a schematic illustration showed the putative binding sites of the miRNAs associated with $3^{\prime} U T R$ of FAM83D. Lower panel: luciferase activity of 3' UTR of Luc-FAM83D in H1299 and H2170 cells transfected with miR-614 mimics or miR-NC. d RNA pull-down assay was used for the detection of FAM83D with either miR-614 or miR-NC probe in H1299 and H2170 cells. e-f Cell proliferation analysis of H1299 and H2170 cells transfected with miR-NC, miR-614, miR-614 + empty vector (EV), or miR-614 + FAM83D by CCK-8 assay. ${ }^{*} P<0.05 ;{ }^{*} P<0.01$ 


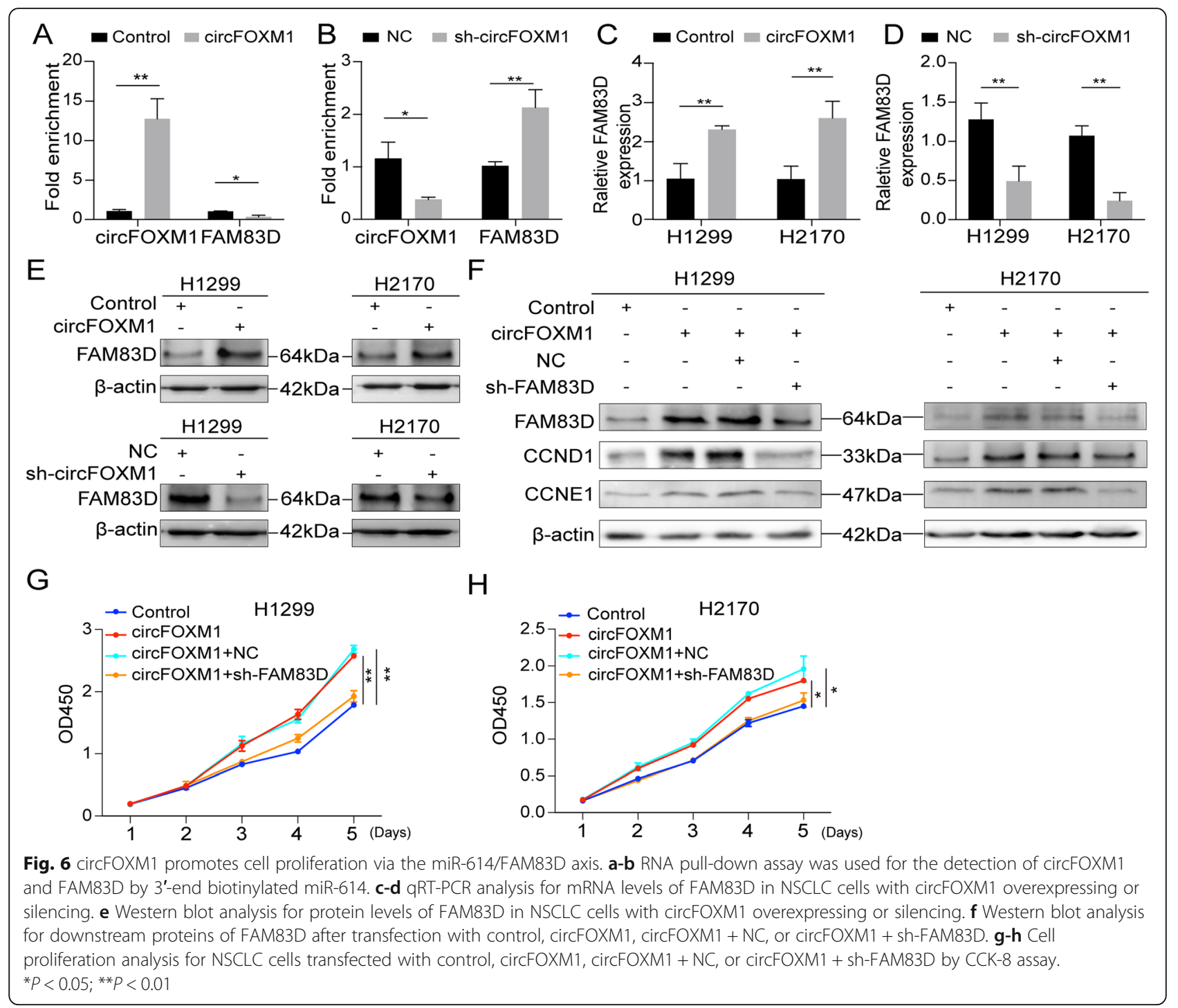

had the opposite effects (Fig. 6c-e). In addition, circFOXM1 expression was positively correlated with FAM83D in clinical NSCLC tissues $(r=0.466, \quad P=$ 0.0008) (Fig. S4H). These evidences indicate that circFOXM1 can function as a miR-614 sponge to promote FAM83D expression.

It has been reported that FAM83D can regulate proteins to affect cell proliferation and cell cycle progressions, such as CCND1 and CCNE1 [16]. As showed in Fig. 6f, overexpression ofcircFOXM1 increased protein levels of CCND1 and CCNE1while silencing FAM83D suppressed the effects of circFOXM1 on upregulation of CCND1 and CCNE1, and their expression levels were decreased. CCK-8 assays also showed that silencing FAM83D decreased the promotion effects of circFOXM1 on cell proliferation (Fig. 6g-h). These results indicate that circFOXM1 promotes cell proliferation of NSCLC cells via the miR-614/FAM83D regulatory axis.

\section{Discussion}

circRNAs are first observed in the 1970s [7]. In the initial studies, the evidence of circRNAs is provided in the viral genetic materials [22-24]. Then, many genes are reported to produce individual circRNAs, such as ETS-1, SRY, and P450 in different cells. However, circRNAs are still considered as rare events with unclear biological functions [25-28]. Until 2012, with the development of high-throughput RNA sequencing and bioinformatics analyses, the landscape of human circRNAs has been disclosed in different cells and tissues [6,29]. Since then, the focus of circRNA research has shifted to elucidate the association with human diseases, including cancers. Serval studies have been reported that the abnormally expressed circRNAs are correlated with clinical features and have the potential to be biomarkers in NSCLC [9, 30, 31]. In this study, we found a circular RNA, has circ_0025039 derived from the exons 4 and 5 of FOXM1 
(named circFOXM1), was obviously upregulated in NSCLC tissues. High expression of circFOXM1 positively correlated with tumor size and advanced stage, and predicted poor prognosis for NSCLC patients.

To date, A large number of studies report that many genes, previously considered as protein-coding genes, can produce circRNAs by back-splicing [32, 33]. Interestingly, it has been described that these circRNAs can be similar or completely different from their maternal gene functions [34-36]. In this study, functional assays showed that circFOXM1 played as oncogenic roles in NSCLC, which is similar to function of its parent gene FOXM1. Liu et al. report that hsa_circ_0025033, another circular RNA derived from the exons 2-10 of FOXM1, also promotes the NSCLC progression [37]. Nowadays, a total of 14 FOXM1-derived circRNAs have been recorded in the circbase database (http://www.circbase. org/) including hsa_circ_0025033, hsa_circ_0025031, hsa_circ_0025035, hsa_circ_0025032, hsa_circ_0025041, hsa_circ_0025039 (circFOXM1), hsa_circ_0025038, hsa_ circ_0025037, hsa_circ_0025042, hsa_circ_0025040, hsa circ_0025030, hsa_circ_009831, hsa_circ_0025036, and hsa_circ_0025034. In our microarray data (GSE126533), 13 circRNAs derived from FOXM1 gene were detected except for hsa_circ_0098318. Among the 13 circRNAs, 5 circRNAs including hsa_circ_0025032, hsa_circ_ 0025038,hsa_circ_0025042,hsa_circ_0025031, and hsa circ_0025039 (circFOXM1) were differential expressed ( $\mid$ Fold Change $\mid>2, P<0.01$ ). Only hsa_circ_0025039 (circFOXM1) was abundant in NSCLC cells (Fig.S6).

The ceRNA hypothesis proposes that RNA transcripts share the same miRNA response elements, resulting in competing for binding to miRNAs, then modulating the expression of each other [38]. In our study, we firstly combined the bioinformatic analyses and circFOXM1 pull-down assays to screen miRNAs, which bind with circFOXM1. Simultaneously, we designed the circFOXM1 luciferase reporter to identify the direct interaction between circFOXM1 and miRNA. We found that miR-614 was the most enriched miRNA in circFOXM1

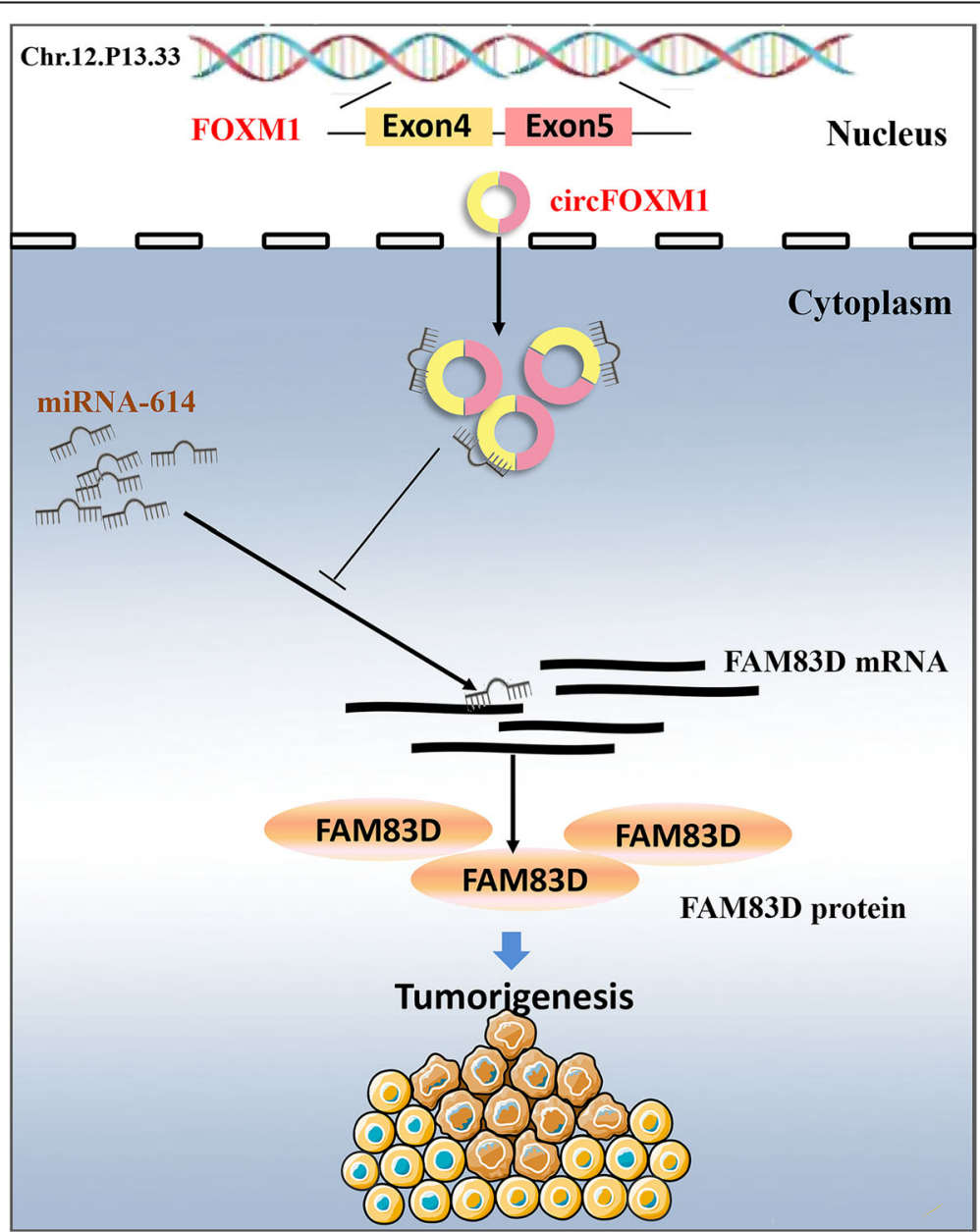

Fig. 7 Schematic diagram of circFOXM1 effects on NSCLC cells proliferation. circFOXM1 acts as a sponge with miR-614 and releases its target FAM83D, resulting in the promoting effect on NSCLC cells proliferation 
pull-down assay, and miR-614 could reduce the luciferase activity of circFOXM1 luciferase reporter by about $50 \%$ (Fig. 4h). However, some miRNAs, such as miR198, miR-516, and miR-6812, could also be enriched in H1299 cells, but not be enriched in H2170 cells. This observation suggests that the mechanism of circFOXM1 may be diverse in different cell lines.

We next analyzed the data of RNA-sequencing and results of miR-614 target prediction, and found FAM83D, a cell-cycle related gene, shared the same miR-614 response elements with circFOXM1. Based on the ceRNA hypothesis, FAM83D should be modulated by circFOXM1/miR-614 ceRNA regulatory network. After a series of experiments, such as RNA pull-down assay, luciferase assay, western blot assay, and qRT-PCR assay, we confirmed that FAM83D was indeed regulated by circFOXM1. Recently, similar mechanisms have been discovered, such as circMOT1/miR-9/p21 regulatory axis [39] and circNRIP1/miR-149/AKT regulatory axis [40].

FAM83D, a microtube associated protein, is reported frequently in various kinds of cancers as an oncogene. So far, FAM83D is considered to be associated with proliferation, migration, and invasion in cancer cells [41]. In NSCLC, it has been reported that FAM83D is a master cell-cycle regulator, resulting in CCND1 and CCNE1 alteration [16]. Although FAM83D function has been studied comprehensively, the regulators of FAM83D are largely unknown. Recently, several studies indicate that FAM83D can be regulated by miRNAs. For example, Yan et al. report that FAM83D is a direct target of miR495 in colorectal cancer cells [14]. He et al. show that FAM83D expression is repressed by miR-210 during cell mitosis [15]. In our study, we first report that FAM83D is directly repressed by miR-614 and it is an essential partner of the circFOXM1 ceRNA regulatory network.

\section{Conclusions}

In summary, our study reveals that circFOXM1 competitively binds to miR-614 to abolish the suppressive effect of miR-614 on FAM83D, and thus promotes cell proliferation (Fig. 7). Our findings provide an insight into understanding the progression of NSCLC and a potential therapeutic biomarker for NSCLC.

\section{Supplementary information}

Supplementary information accompanies this paper at https://doi.org/10. 1186/s13046-020-01555-5.

Additional file 1: Table S1. The main primers used in this study. Additional file 2: Table S2. The all 685 differently expressed genes in H1299 cells after silencing circFOXM1.

Additional file 3: Table S3. Predicted 54 mRNAs targeting by miR614(8 mer sites).
Additional file 4: Figure S1. Overexpressing or silencing circFOXM1 could not disturb the expression of FOXM1. (A)Kaplan-Meier survival analysis of circFOXM1 expression in NSCLC patients. (B) Expression of circFOXM1 in1 normal cell line (a human bronchial epithelial cell line BEAS-2B) and 4 NSCLC cell lines. (C) Schematic view for construction of circFOXM1 overexpression vector. (D) Schematic view for sh-circFOXM1 targeted site. (E) mRNA levels of circFOXM1 and FOXM1 in H1299 and H2170 cells after transduction with circFOXM1 overexpression vector. (F) mRNA levels of circFOXM1 and FOXM1 in H1299 and H2170 cells after transduction with circFOXM1 shRNA. (G) Protein levels of FOXM1 in H1299 and H2170 cells with circFOXM1 overexpression. (H) Protein levels of FOXM1 in H1299 and H2170 cells with circFOXM1 knockdown. ${ }^{*} P<$ $0.05 ;{ }^{*} P<0.01$.

Additional file 5: Figure S2. circFOXM1 mutation could not promote NSCLC cells proliferation and cell cycle progression. (A) Western blot analysis for Flag-Ago2 or Flag-tag expression. (B) CCK-8 assay was performed to determine the viability in $\mathrm{H} 1299$ and $\mathrm{H} 2170$ cells with overexpressing circFOXM1 mutation (circFOXM1 mut). (C) Flow cytometry analysis showed no obvious alteration in the proportion of cell in G1 phase and G2/M phase in H1299 and H2170 cells with overexpressing cirCFOXM1 mut.

Additional file 6: Figure S3. miR-614 inhibits cell proliferation. (A-B) CCK-8 and colony formation assays were performed to determine the viability in $\mathrm{H} 1299$ and H2170 cells after transfecting with miR-614 mimics. (C-D) Flow cytometry analysis showed increase in the proportion of cell in G1 phase and decrease in the proportion of cell in S and G2/M phase after transfecting with miR-614 mimics. (E) Expression of miR-614 was significantly downregulated in the 5 paired samples of NSCLC tissues by analysis in our GSE126533 data. T, tumor tissue; N, nontumor tissue. (F) Expression of miR-614 was examined in 20 paired samples of NSCLC. U6 was used as control. (G) Kaplan-Meier survival analysis of miR-614 expression in NSCLC patients by analysis of Kaplan Meier-plotter database. ${ }^{*} P<$ $0.05 ;{ }^{* *} P<0.01$.

Additional file 7: Figure S4. Silencing FAM83D inhibits cell proliferation. (A-B) CCK-8 and colony formation assays were performed to determine the viability in $\mathrm{H} 1299$ and H2170 cells with silencing FAM83D. (C-D) Flow cytometry analysis showed an alteration in the proportion of cell in G1 phase and G2/M phase in NSCLC cells with FAM83D silencing. (E) Expression of FAM83D was significantly upregulated in the 5 paired samples of NSCLC tissues by analysis in our GSE126533 data. (F) Expression analysis for FAM83D mRNA in TCGA database. (G) Kaplan-Meier survival analysis of FAM83D expression in NSCLC patients by analysis of Kaplan Meier-plotter database. $(H)$ Correlation analysis of circFOXM1 and FAM83D in NSCLC tissues. ${ }^{*} P<0.05 ;{ }^{* *} P<0.01$.

Additional file 8: Figure S5. Absolute quantification for circFOXM1 and FAM83D. Absolute quantification for circFOXM1 and FAM83D mRNA in H1299 and H2170 cells.

Additional file 9: Figure S6. Expression of circRNAs derived from FOXM1 in NSCLC cells. Relative expression of hsa_circ_0025039 (circFOXM1), hsa_circ_0025038, hsa_circ_0025042, hsa_circ_0025031, and hsa_circ_0025032 in NSCLC cells. ${ }^{* *} P<0.01 ;{ }^{* * *} P<0.001$.

\section{Abbreviations}

NSCLC: Non-small cell lung cancer; circRNAs: Circular RNAs; miRNA: MicroRNA; FAM83D: Family with sequence similarity 83-member D; Cyclin D1: CCND1; Cyclin E1: CCNE1; CCK-8: Cell Counting Kit-8;

FISH: Fluorescence in situ hybridization

\section{Acknowledgements}

Not applicable.

\section{Authors' contributions}

WQ supported and supervised the study. LJ collected the NSCLC tissues and supervised the study. CY, ZC and SC performed all the experimental validation assays and wrote the manuscript. XM, BL, YF, HW, HJ, QY and XZ analyzed data. The author(s) read and approved the final manuscript. 


\section{Funding}

This work was supported by grants from National Natural Science Foundation of China (81772461, 81402278), National Key Research and Development Program of China (No.2017YFC0907905, No.2018YFC1313600), Shanghai Municipal Commission of Health and Family Planning (No.17411971100), and State Key Laboratory of Oncogenes and Related Gene (91-17-12).

\section{Availability of data and materials}

The datasets used and analyzed in the current study are available from the corresponding author on reasonable request.

\section{Ethics approval and consent to participate}

This study was approved by the Ethics Committees of the Shanghai Chest Hospital, Shanghai Jiao Tong University and the Shanghai Medical Experimental Animal Care Commission.

\section{Consent for publication}

All authors have agreed to publish this manuscript.

\section{Competing interests}

The authors declare that they have no competing interests.

\section{Author details}

${ }^{1}$ State Key Laboratory of Oncogenes and Related Genes, Shanghai Cance Institute, Renji Hospital, Shanghai Jiao Tong University School of Biomedical Engineering, Shanghai 200032, China. ${ }^{2}$ Department of Respiratory Medicine, Shanghai Chest Hospital, Shanghai Jiao Tong University, Shanghai 200030, China. ${ }^{3}$ Department of Thoracic Surgery, Renji Hospital, Shanghai Jiao Tong University School of Medicine, Shanghai 200032, China. ${ }^{4}$ Shanghai Cancer Institute, Renji Hospital, Shanghai Jiao Tong University School of Medicine, Shanghai 200032, China.

\section{Received: 20 December 2019 Accepted: 8 March 2020}

\section{Published online: 30 March 2020}

\section{References}

1. Bray F, Ferlay J, Soerjomataram I, Siegel RL, Torre LA, Jemal A. Global cancer statistics 2018: GLOBOCAN estimates of incidence and mortality worldwide for 36 cancers in 185 countries. CA Cancer J Clin. 2018;68(6):394-424.

2. Goldstraw P, Ball D, Jett JR, Le Chevalier T, Lim E, Nicholson AG, Shepherd FA. Non-small-cell lung cancer. Lancet. 2011;378(9804):1727-40.

3. Hirsch FR, Scagliotti GV, Mulshine JL, Kwon R, Curran WJ Jr, Wu YL, Paz-Ares L. Lung cancer: current therapies and new targeted treatments. Lancet. 2017;389(10066):299-311.

4. Vecchiarelli S, Bennati C. Oncogene addicted non-small-cell lung cancer: current standard and hot topics. Future Oncol. 2018;14(13s):3-17.

5. Rotow J, Bivona TG. Understanding and targeting resistance mechanisms in NSCLC. Nat Rev Cancer. 2017;17(11):637-58.

6. Djebali S, Davis CA, Merkel A, Dobin A, Lassmann T, Mortazavi A, Tanzer A, Lagarde J, Lin W, Schlesinger F, et al. Landscape of transcription in human cells. Nature. 2012;489(7414):101-8.

7. Hsu MT, Coca-Prados M. Electron microscopic evidence for the circular form of RNA in the cytoplasm of eukaryotic cells. Nature. 1979;280(5720):339-40

8. Danan M, Schwartz S, Edelheit S, Sorek R. Transcriptome-wide discovery of circular RNAs in Archaea. Nucleic Acids Res. 2012;40(7):3131-42.

9. Di X, Jin X, Li R, Zhao M, Wang K. CircRNAs and lung cancer: Biomarkers and master regulators. Life Sci. 2019;220:177-85.

10. Cheng Z, Yu C, Cui S, Wang H, Jin H, Wang C, Li B, Qin M, Yang C, He J, et al. circTP63 functions as a ceRNA to promote lung squamous cell carcinoma progression by upregulating FOXM1. Nat Commun. 2019;10(1): 3200.

11. Qiu M, Xia W, Chen R, Wang S, Xu Y, Ma Z, Xu W, Zhang E, Wang J, Fang T, et al. The Circular RNA circPRKCI Promotes Tumor Growth in Lung Adenocarcinoma. Cancer Res. 2018;78(11):2839-51.

12. Wang F, Zhang S, Wei Y, Chen H, Jiao Z, Li Y. Upregulation of family with sequence similarity 83 member $D$ expression enhances cell proliferation and motility via activation of Wnt/beta-catenin signaling and predicts poor prognosis in gastric cancer. Cancer Manag Res. 2019;11:6775-91.
13. Liao W, Liu W, Liu X, Yuan Q, Ou Y, Qi Y, Huang W, Wang Y, Huang J. Upregulation of FAM83D affects the proliferation and invasion of hepatocellular carcinoma. Oncotarget. 2015;6(27):24132-47.

14. Yan L, Yao J. Qiu J: miRNA-495 suppresses proliferation and migration of colorectal cancer cells by targeting FAM83D. Biomed Pharmacother. 2017; 96:974-81.

15. He J, Wu J, Xu N, Xie W, Li M, Li J, Jiang Y, Yang BB, Zhang Y. MiR-210 disturbs mitotic progression through regulating a group of mitosis-related genes. Nucleic Acids Res. 2013;41(1):498-508.

16. Shi R, Sun J, Sun Q, Zhang Q, Xia W, Dong G, Wang A, Jiang F, Xu L. Upregulation of FAM83D promotes malignant phenotypes of lung adenocarcinoma by regulating cell cycle. Am J Cancer Res. 2016;6(11):258798.

17. Zhang XO, Wang HB, Zhang Y, Lu X, Chen LL, Yang L. Complementary sequence-mediated exon circularization. Cell. 2014;159(1):134-47.

18. Thomson DW, Dinger ME. Endogenous microRNA sponges: evidence and controversy. Nat Rev Genet. 2016;17(5):272-83.

19. Tay Y, Rinn J, Pandolfi PP. The multilayered complexity of ceRNA crosstalk and competition. Nature. 2014;505(7483):344-52.

20. Gyorffy B, Surowiak P, Budczies J, Lanczky A. Online survival analysis software to assess the prognostic value of biomarkers using transcriptomic data in non-small-cell lung cancer. PLoS One. 2013;8(12):e82241.

21. Agarwal V, Bell GW, Nam JW, Bartel DP. Predicting effective microRNA target sites in mammalian mRNAs. eLife. 2015;4:e05005.

22. Kolakofsky D. Isolation and characterization of Sendai virus DI-RNAs. Cell. 1976;8(4):547-55.

23. Hewlett MJ, Pettersson RF, Baltimore D. Circular forms of Uukuniemi virion RNA: an electron microscopic study. J Virol. 1977;21(3):1085-93.

24. Kos A, Dijkema R, Arnberg AC, van der Meide PH, Schellekens H. The hepatitis delta (delta) virus possesses a circular RNA. Nature. 1986;323(6088): 558-60

25. Capel B, Swain A, Nicolis S, Hacker A, Walter M, Koopman P, Goodfellow P, Lovell-Badge R. Circular transcripts of the testis-determining gene Sry in adult mouse testis. Cell. 1993;73(5):1019-30.

26. Cocquerelle C, Mascrez B, Hetuin D, Bailleul B. Mis-splicing yields circular RNA molecules. FASEB J. 1993;7(1):155-60.

27. Zaphiropoulos PG. Exon skipping and circular RNA formation in transcripts of the human cytochrome P-450 2C18 gene in epidermis and of the rat androgen binding protein gene in testis. Mol Cell Biol. 1997;17(6):2985-93.

28. Zaphiropoulos PG. Circular RNAs from transcripts of the rat cytochrome P450 2C24 gene: correlation with exon skipping. Proc Natl Acad Sci U S A. 1996:93(13):6536-41.

29. Salzman J, Gawad C, Wang PL, Lacayo N, Brown PO. Circular RNAs are the predominant transcript isoform from hundreds of human genes in diverse cell types. PLoS One. 2012;7(2):e30733.

30. He J, Xie Q, Xu H, Li J, Li Y. Circular RNAs and cancer. Cancer Lett. 2017;396: 138-44.

31. Kristensen LS, Hansen TB, Veno MT, Kjems J. Circular RNAs in cancer: opportunities and challenges in the field. Oncogene. 2018;37(5):555-65.

32. Zhao $X$, Cai $Y, X u$ J. Circular RNAs: biogenesis, mechanism, and function in human cancers. Int J Mol Sci. 2019;20(16):3926.

33. Holdt LM, Kohlmaier A, Teupser D. Molecular roles and function of circular RNAs in eukaryotic cells. Cell Mol Life Sci. 2018;75(6):1071-98.

34. Xiao-Long M, Kun-Peng Z, Chun-Lin Z. Circular RNA circ_HIPK3 is downregulated and suppresses cell proliferation, migration and invasion in osteosarcoma. J Cancer. 2018;9(10):1856-62.

35. Zheng Q, Bao C, Guo W, Li S, Chen J, Chen B, Luo Y, Lyu D, Li Y, Shi G, et al. Circular RNA profiling reveals an abundant circHIPK3 that regulates cell growth by sponging multiple miRNAs. Nat Commun. 2016;7:11215.

36. Liu Y, Qian L, Yang J, Huang H, Feng J, Li X, Bian T, Ke H, Liu J, Zhang J. The expression level and prognostic value of HIPK3 among non-small-cell lung cancer patients in China. Onco Targets Ther. 2018;11:7459-69.

37. Pan Y, Xu T, Liu Y, Li W, Zhang W. Upregulated circular RNA circ_0025033 promotes papillary thyroid cancer cell proliferation and invasion via sponging miR-1231 and miR-1304. Biochem Biophys Res Commun. 2019; 510(2):334-8.

38. Salmena L, Poliseno L, Tay $Y$, Kats L, Pandolfi PP. A ceRNA hypothesis: the Rosetta stone of a hidden RNA language? Cell. 2011;146(3):353-8.

39. Han D, Li J, Wang H, Su X, Hou J, Gu Y, Qian C, Lin Y, Liu X, Huang M, et al. Circular RNA circMTO1 acts as the sponge of microRNA-9 to suppress hepatocellular carcinoma progression. Hepatology. 2017;66(4):1151-64. 
40. Zhang X, Wang S, Wang H, Cao J, Huang X, Chen Z, Xu P, Sun G, Xu J, Lv J, et al. Circular RNA circNRIP1 acts as a microRNA-149-5p sponge to promote gastric cancer progression via the AKT1/mTOR pathway. Mol Cancer. 2019; 18(1):20.

41. Huang M, Ma X, Shi H, Hu L, Fan Z, Pang L, Zhu F, Yang X, Xu W, Liu B, et al. FAM83D, a microtubule-associated protein, promotes tumor growth and progression of human gastric cancer. Oncotarget. 2017;8(43):74479-93.

\section{Publisher's Note}

Springer Nature remains neutral with regard to jurisdictional claims in published maps and institutional affiliations.

Ready to submit your research? Choose BMC and benefit from:

- fast, convenient online submission

- thorough peer review by experienced researchers in your field

- rapid publication on acceptance

- support for research data, including large and complex data types

- gold Open Access which fosters wider collaboration and increased citations

- maximum visibility for your research: over $100 \mathrm{M}$ website views per year

At $B M C$, research is always in progress.

Learn more biomedcentral.com/submissions 